\title{
Relación entre precios relativos de bienes y exportaciones periodo 2010 - 2014
}

\section{Relationship between relative prices of goods and exports period 2010 - 2014}

\author{
Sebastián Cárdenas Zambrano \\ Mónica Clavijo Basantes \\ Martha Paola Alvarado Vera \\ Universidad de Especialidades Espíritu Santo, Ecuador
}

Autor para correspondencia: scardenasz@uees.edu.ec, monicaclavijo@uees.edu.ec, malvarve@uees.edu.ec

Fecha de recepción: 10 de septiembre de 2017 - Fecha de aceptación: 30 de noviembre de 2017

\section{Resumen}

El trabajo de investigación pretendió demostrar que el sector exportador en el Ecuador para el periodo 2010-2014 guarda relación con los precios relativos de los bienes. Esta relación se explicó efectuando el modelo clásico de exportaciones planteado por Mesa, Cock y Jiménez (1999), la cual pone en consideración los precios relativos de bienes y la competitividad de la industria. Para validar la hipótesis del modelo, se utilizó la herramienta estadística de regresión linear múltiple usando las variables: tasa de cambio real, salario real e índice de precio al productor. Con un $\mathrm{R}$ cuadrado ajustado de 0,8625 , se comprobó que las variables utilizadas para el análisis tienen estrecha relación con las exportaciones ecuatorianas. Los resultados del estudio demuestran que un mayor incentivo en las variables empleadas tendrá un efecto positivo en las exportaciones, razón por la cual es necesario mejorar las condiciones para el mejor desempeño de las exportaciones.

Palabras clave: exportaciones; tipo de cambio; índice de precios al productor; salarios; ecuador

\begin{abstract}
The research sought to demonstrate that the export sector in Ecuador for the period 2010-2014 is related to the relative prices of goods. This relationship is explained by making the export model proposed by Mesa, Cock and Jimenez (1999), which puts into consideration the relative prices of goods and the competitiveness of the industry. To validate the hypothesis, the statistical tool was used multiple linear regression using the variables: real exchange rate, real wages and producer price index. With an adjusted R-squared of 0,8625, it was found that the variables used in the analysis are closely related to Ecuador's exports. The study results show that a greater incentive in the variables used will have a positive effect on exports, which is why it is necessary to improve the conditions for better export performance.
\end{abstract}

Key words: exports; exchange rate; index of producer prices; wages; Ecuador 


\section{Introducción}

Las actividades comerciales se han llevado a cabo por los seres humanos durante siglos. En la actualidad gracias a la globalización se vuelve de vital importancia la interacción comercial entre los diferentes países (Berdún Chéliz P., 2002). La exportación a un mercado extranjero es una estrategia de muchos países dado que algunos países no ofrecen una suficiente oportunidad para justificar la producción local, la exportación permite la fabricación de productos especializados dentro de un mercado y de esa manera ofrecer en el ámbito internacional el bien producido y así también obtener economías de escala.

La economía mundial ha cambiado sustancialmente en la última década, con profundas implicaciones respecto a los países con las condiciones de competitividad y las estructuras geográficas y sectoriales del comercio mundial (Cardozo, Chavarro, \& Ramírez, 2007). La globalización de la economía ha conseguido acentuar la relación con el comercio internacional, expresada en la cada vez mayor dependencia entre los países con un aumento en la intensidad de las transacciones comerciales internacionales.

Asimismo, el creciente desarrollo de las exportaciones en el comercio internacional ha tenido una influencia en el comportamiento en los precios ya que ante las diferentes condiciones que los países enfrentan se permite que los productos se mantengan con costos de producción muy diferenciados (Balaguer, Orts, \& Uriel, 2000). Es donde radica una de las múltiples ventajas que los países pueden adquirir frente a otros países, su costo de producción, el cual se le ha prestado mucha atención en los últimos años.

El comercio internacional es uno de los ejes fundamentales para el desarrollo de las actividades económicas del Ecuador. Tradicionalmente el país se ha caracterizado por ser un exportador de productos primarios como es en el caso del cacao, el cual fue el principal producto exportado hasta el año 1920, convirtiéndose el ecuador en el mayor exportador mundial de cacao durante los años de 1880 a 1915 (Uquillas, 2008).

De acuerdo con lo planteado por Báez (2014) a partir de los 50s Ecuador se integra al mercado mundial a través de la exportación de banano, el cual se convirtió en el eje de la economía del país hasta la década de los 60s. Desde esta fecha hasta la actualidad, el petróleo y sus derivados constituyen el principal producto de exportación, representando en un $66 \%$ aproximadamente del total de las exportaciones.

Si se considera que el crecimiento económico se alcanza en base al mayor grado de la diversificación de las exportaciones (Bajo Rubio, 1996), y ya que la economía ecuatoriana ha estado sujeto a la exportación de pocos productos especialmente primarios, en los últimos años se ha procurado la inclusión en nuevos mercados de productos no tradicionales para conseguir una oferta exportable compleja.

A pesar de que un gran porcentaje de las exportaciones ecuatorianas corresponde al petróleo, se registra una significativa variación en el monto de las exportaciones no petroleras (Báez P, 2014), lo que permite conjeturar que existen factores importantes que determinan este comportamiento en las exportaciones ecuatorianas. Por lo tanto, es fundamental analizar al sector 
exportador de manera más específica, puesto que los estudios realizados previamente consideran solo ciertos aspectos de esta actividad son determinar la influencia que tiene la venta internacional en el desarrollo económico del país ni los principales factores que determinan la exportación, por lo que la problemática planteada por este artículo es conocer en qué medida los precios relativos de los bienes influye en el comportamiento de las exportaciones.

\section{Marco Teórico}

La teoría de ventaja comparativa de David Ricardo instituye que el comercio internacional no pretende ventajas absolutas diferentes y que es posible entablar una relación comercial cuando existen ventajas comparativas, las cuales estarán presente siempre que exista una diferencia en los requerimientos relativos de trabajo provocando que el costo de oportunidad doméstico de dos bienes sea distinto en dos naciones (Blanco, 2011).

Blanco (2011) dice que la teoría Ricardiana establece que continuamente existirá comercio internacional siempre y cuando se presente una diferente clase de especialización relativa entre dos bienes de diferentes países.

La teoría de David Ricardo es una de las más aceptadas entre los economistas, sin embargo, a menudo es confundida con la teoría de ventaja absoluta de Adam Smith. La teoría ricardiana se basa en la comparación de costos relativos o costos comparativos (Bajo Rubio, 1996), estableciendo que todos los países se benefician del comercio internacional si se dedican a la producción de bienes que pueden elaborar bajo un costo relativo menor.

El costo de oportunidad es constante para el modelo de ventaja comparativa; por tanto, representa para un país elegir entre incrementar la producción de un bien en el que tiene ventaja comparativa y sacrificar la producción del otro bien (De la Rosa Mendoza, 2006). Esto se puede evidenciar a través de la frontera de posibilidades de producción, que en el caso del modelo ricardiano es una línea recta, porque considera solamente un factor de producción, el trabajo. Por lo que Balaguer, Orts y Uriel (2000) expresan que, si el costo de oportunidad es menor que el precio relativo de un bien, el país se especializará en la producción de dicho bien.

Según Díaz-Alejandro (1977) la evidencia empírica muestra que a pesar de que el modelo ricardiano no describe totalmente las causas y consecuencias del comercio mundial, la mayoría de países se adaptan a la base de este modelo; ya que, exportan bienes en los que su productividad relativa es más alta e importan aquellos bienes que producen de manera relativamente menos eficiente, es decir, adquieren bienes que implican un costo mayor al momento de producirlos domésticamente.

Conjuntamente, Blanco (2011) expone el modelo de Hecksher-Ohlin conocido también como teoría de las proporciones factoriales establece que si un país tiene abundancia relativa de un factor sea trabajo o capital, tendrá una ventaja comparativa y por lo tanto exportará aquellos bienes que requieran mayor cantidad de este bien.

Cabe recalcar que el modelo Hecksher-Ohlin es un complemento de la teoría de David Ricardo, considera la cantidad y la disposición de un factor para definir la especialidad 
productiva, además en este modelo se añade el factor capital como determinante para la productividad de los países.

Los aportes realizados por los economistas Hecksher y Ohlin a la teoría del comercio internacional fueron desarrollados en base a la teoría de David Ricardo. Esta teoría establece que la ventaja comparativa surge de la diferencia en las dotaciones de factores entre diferentes países, es decir, un país exportará aquel bien que tenga mayor cantidad del factor que le es relativamente abundante (Davis, 1995).

El modelo H-O refleja que el comercio internacional no solo está determinado por la productividad del trabajo sino también por la diferencia de recursos que existe entre los países.

Ningún país es igual a otro, todos poseen diferencias en cuanto a la dotación de factores y es esta diferencia la que permite la existencia del comercio internacional. Debido a la distinta dotación de factores, un bien puede producirse en un país con menor costo que en otro.

Sin comercio internacional; es decir en condiciones de autarquía, Morón Cárdenas (2002) indica que el consumo de un país está limitado por su frontera de posibilidades de producción doméstica del mismo.

Con comercio internacional se puede ampliar las posibilidades de elección entre viene, ya que, permite combinaciones de consumo que no se alcanzarían solo con la producción doméstica. El consumidor alcanza una curva de indiferencia más alta, pues puede elegir entre canastas antes inexistente que se hallaban fuera de las posibilidades de producción de su país. Entonces participando en el mercado mundial, el país alcanza un grado de bienestar más alto de lo que hubiera alcanzado en autarquía.

Las ganancias del comercio internacional están determinadas también como señala Zhang y Liu (2012), por los precios relativos a lo que un país se expone cuando participa en el mercado mundial, cuando existe diferencia entre el precio relativo de un bien en el país doméstico y el precio relativo internacional. Entonces el país domestico será más eficiente en la producción de dicho bien en el cual es relativamente menos eficiente, ya que gracias al comercio internacional puede intercambiar unidades del bien que produce de manera relativamente eficiente por unidades del bien en el que no posee ventaja comparativa. Esto significa que la importación constituye una producción indirecta del bien con desventaja comparativa, permitiéndole al país disminuir los costos en lo que incurriría al fabricar este bien. Además, se puede decir que los países obtendrán una suma final de bienes mayor que la que hubiera obtenido si cada país producía todos los bienes.

Si se considera el modelo Hecksher-Ohlin, se encuentra otra ventaja de participar en el comercio internacional y esta es el incremento de la demanda en el extranjero del factor relativamente abundante en el país. Si bien los factores de producción no son consumidos de manera directa son utilizados en la producción de bienes finales, entonces el país extranjero compra indirectamente el bien que le es relativamente escaso a través de la importación de bienes que utilicen mayormente este factor. 
Es así que la globalización permite el desarrollo del comercio internacional entre todos los países que decidan participar, sin embargo, a pesar de los avances de la globalización cada país o grupo de países guardan características que los diferencian unos de otros como el idioma, costumbres, religión e incluso la moneda, que es donde surge la diferencia entre los precios de los bienes.

Esta diferencia a lo largo de múltiples investigaciones ha sido evaluada mediante la variable del tipo de cambio. Asiain (2010) plantea al tipo de cambio como el precio de una moneda en términos de otra, también se puede decir que, es el precio al cual la divisa de un país puede ser convertida a la divisa de otro país. El tipo de cambio está determinado en el mercado de divisas y depende de varios factores como la oferta, la inflación, la demanda, entre otros. El tipo de cambio mide el grado de competitividad de los productos nacionales respecto a productos extranjeros y se refiere al precio relativo de los bienes y servicios de un país. La importancia de los tipos de cambio radica en que sirve a las empresas, gobiernos y particulares para convertir los precios extranjeros en sus respectivos precios en moneda nacional. Los tipos de cambio desempeñan un papel fundamental en el comercio internacional, al permitir la comparación de los precios de bienes locales versus bienes extranjeros.

Aller, Sáinz y Sáenz (2002) consideran la dinámica del comercio internacional, y concluyen que el tipo de cambio juega un papel importante, los consumidores nacionales encuentran a las importaciones del extranjero más baratas, si la moneda local se aprecia respecto de la extranjera, además incrementa el precio relativo de las exportaciones nacionales disminuyendo su volumen, por ende reduciendo el nivel de competitividad de los productos locales en el mercado internacional; lo contrario si a moneda se deprecia.

El comercio exterior es una actividad fundamental en la vida económica de todos los países, marcando la prosperidad o declive de la economía. Tradicionalmente Ecuador se ha caracterizado por ser un país exportador de bienes primarios. Y a lo largo de los años ha venido evolucionando con respecto a la actividad exportadora.

Ecuador posee características particulares en cuanto a clima, ubicación geográfica, dotación de recursos, temperatura, entre otras características del entorno ambiental y económico que le otorgan una notable ventaja comparativa al momento de producir bienes. Es por esta razón que para el desarrollo de este trabajo de investigación se ha tomado como premisa principal la teoría de la ventaja comparativa de David Ricardo.

Ecuador representa una pequeña economía abierta, cuyo volumen de exportaciones depende de las condiciones internas de producción, mientras que los precios de los productos exportables se fijan en el mercado internacional por lo que se toma como base un modelo reducido de exportaciones desarrollado por Mesa, Cock y Jiménez (1999) que incorpora supuestos que se ajustan a la situación ecuatoriana y que además permite incorporar el modelo de David Ricardo al caso ecuatoriano.

En el modelo de Mesa, Cock y Jiménez (1999) se plantean funciones de oferta y demanda bajo los siguientes supuestos: 1) la economía es pequeña frente a otros mercados, 2) la demanda es elástica, y 3) los empresarios son tomadores de precios. 
Al suponer una economía pequeña y abierta, los precios de los bienes que se comercializan son fijados en el mercado mundial lo que provoca que las economías con estas características enfrenten una curva de demanda externa infinitamente elástica, además el volumen de exportaciones dependerá de las condiciones internas de producción.

El modelo general de las exportaciones obtenido por Mesa, Cock y Jiménez (1999) consideran que la oferta real de exportaciones y demanda real de exportaciones son iguales en el equilibrio, además expresan las ecuaciones de oferta y demanda reales en función del precio externo de venta de los productos que exporta el país y formulan el modelo reducido de la siguiente manera:

$$
\ln X_{t}=A_{1} \ln \left[\frac{T_{e i} P_{e t}}{P_{e t}}\right]+A_{2} \ln Y_{e}+A_{3} \ln Y^{*}{ }_{i}
$$

Mesa, Cock y Jiménez (1999) se refieren al término $\left[\frac{T_{e i} P_{e t}}{P_{e t}}\right]$ como la tasa de cambio real de las exportaciones. Además, $A_{0}, A_{1}, A_{2}, A_{3}$ son números positivos.

Está ecuación representa al modelo reducido de exportaciones en una situación de equilibrio, bajo el supuesto de competencia perfecta y que el mercado de exportaciones se encuentra en equilibrio, lo que permite igualar ecuaciones de oferta y demanda de exportaciones. Mediante la ecuación se expresa como una función de los precios relativos de bienes y la competitividad de la industria.

Justamente en varias investigaciones empíricas se ha visto la necesidad de indagar acerca de los diferentes factores determinantes de las exportaciones dando como resultado la evaluación de varias variables, por ejemplo, Doherty (2012) en su estudio mediante la construcción de tres medidas que indiquen el nivel de la globalización de la industria y la comparación de esos valores con los cambios de precios, estableció que la relación entre la variación de los precios de la industria y los niveles de comercialización global fue significativa en el período 1997 y 2002 en los Estados Unidos; sin embargo, entre la intensidad de la exportación y el cambio de los precios internos, la relación correspondiente no fue consistente.

Sin embargo, en la investigación de Camacho (2011) en la cual se estiman dos modelos estacionarios de regresión múltiple donde en el primero se toma en cuenta la productividad laboral, el tipo de cambio real, los salarios, variaciones de precios, entre otras variables; expone la importancia que tienen las variaciones en las variables de precios para el crecimiento en lo que respecta a las exportaciones manufactureras mexicanas.

Por otro lado, Gómez y Camacho (2011) aplicando el modelo de co-integración propuesta por Engle Granger, presenta que existe una relación de equilibrio, a largo plazo, entre las exportaciones y la productividad de la mano de obra. Además, Cuevas-Ahumada (2011) realiza una comparación entre las determinantes de las exportaciones manufactureras de Argentina y México donde se revela un hecho importante. En ambas naciones tanto la productividad laboral, la demanda externa y precios de las industrias influyen en las exportaciones. 


\section{Metodología}

Esta investigación se desarrolló bajo un enfoque positivista aplicando el método deductivo. Además, se trabajó en el enfoque cuantitativo de forma no experimental ya que se obtuvo la información de fuentes secundarias. El trabajo tuvo un horizonte de estudio longitudinal ya que se tomó en cuenta los datos del periodo 2010-2014.

El trabajo de investigación pretendió demostrar que el sector exportador en el Ecuador para el periodo 2010-2014 guarda relación con los precios relativos de los bienes. Esta relación se intentó explicar efectuando el modelo de exportaciones planteado por Mesa, Cock y Jiménez (1999), la cual pone en consideración los precios relativos de bienes y la competitividad de la industria.

Para la ecuación planteada se estimó que los precios relativos de los bienes es el índice del tipo de cambio real el cual se puede definir como la relación ente el nivel de precios de la economía interna y el nivel de precios externos, expresados en una única moneda. Y en la dimensión de la competitividad de la industria ecuatoriana se utilizó como variables los salarios reales y el Índice de Precios al Productor. Las exportaciones se vieron reflejadas en el total de exportaciones con valores FOB publicadas por el Banco Central.

Para validar la hipótesis planteada, se utilizó la herramienta estadística de regresión linear múltiple de cointegración usando las variables: tasa de cambio real, salario real e índice de precio al productor. Se utilizaron datos provenientes del Banco Central en una medida mensual en el periodo 2010-2014. Dado que los datos se encontraron en años bases diferentes se procedió a plantearlos en un único año base, estableciendo como año base el 2007. Además, las variables fueron previamente transformadas en logaritmos para facilitar su interpretación como elasticidades.

Con esta aplicación estadística se intentó demostrar que existe evidencia para aceptar la premisa que existe relación entre el precio relativo de bienes y las exportaciones. El objetivo del análisis de la regresión aplicada fue examinar cómo el modelo pretende explicar el comportamiento de la variable dependiente que en este caso es la variable "Exportaciones" (EXP), que se denotó por Y, utilizando la información proporcionada por los valores de las variables independientes consideradas las cuales son: "Índice de Precios al Productor" (IPP), “Tipo de Cambio Real” (TC), y "Salario Real” (SR).

\section{Resultados}

La presente investigación abarca la información mensual del periodo 2000-2014, las variables empleadas para el desarrollo del modelo fueran el monto de las exportaciones (EXP), el tipo de cambio real (TCR), los salarios reales (SR) y el índice de precios al productor (IPP).

Las series consideradas para el análisis constan de un total de 60 observaciones que comprenden el periodo de estudio. Para esta sección se utiliza el programa estadístico EViews. 
Para la validación de los datos se realizó una prueba de estacionariedad. Se validó que ninguna de las series es estacionaria, ya que ninguna presenta una media constante a lo largo del período considerado.

Para comprobar la evidencia de no estacionariedad, se realiza el correlograma correspondiente a cada una de las variables, el mismo que indica una potencial presencia de raíz unitaria. Se evidencia en la prueba que el primer coeficiente de autocorrelación parcial es cercano a 1 siendo los coeficientes restantes cercanos a cero para todos los casos, lo que indica que la serie se puede diferenciar para hacerla estacionaria.

Como primer punto, se grafica las series en nivel. En el gráfico \#1, se observa que ninguna de las series es estacionaria; ya que ninguna presenta una media constante a lo largo del periodo considerado.
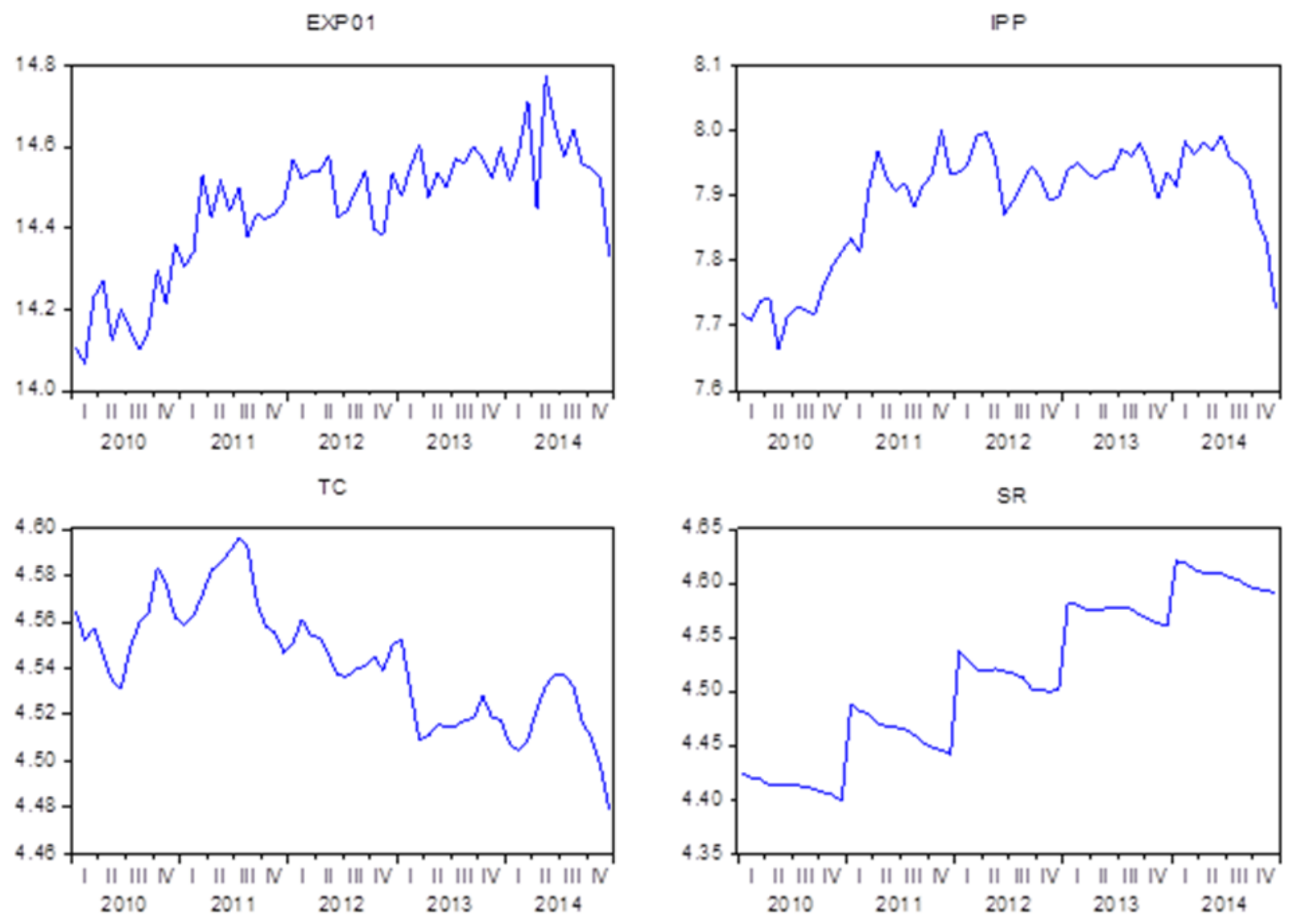

Grafico \#1: Comportamiento de las series EXP, IPP, SR y TCR.

La Tabla \# 1 muestra los resultados al aplicar el modelo planteado bajo el método de mínimos cuadrados. Se ajustó la muestra a 59 observaciones para el empleo del modelo. Se comprueba que las tres variables independientes efectivamente tienen relación positiva con la variable dependiente. El R cuadrado ajustado nos permite conocer que mencionada relación expresada en porcentaje nos representaría un $86,25 \%$ de relación entre las variables estipuladas en el modelo. 
Tabla\# 1: Modelo de Mínimos Cuadrados para las Exportaciones.

\begin{tabular}{crllr}
\hline Variable & $\begin{array}{rlll}\text { Coefficien } \\
\mathrm{t}\end{array}$ & Std. Error & t-Statistic & Prob. \\
\hline IPP & 0.969905 & 0.120773 & 8.030802 & 0.0000 \\
TC & 0.430178 & 0.127613 & 3.370966 & 0.0014 \\
SR & 1.073068 & 0.139910 & 7.669705 & 0.0000 \\
RESID01(-1) & -0.352192 & 0.125115 & -2.814951 & 0.0068 \\
R-squared & 0.869640 & Mean dependent var & 14.45321 \\
Adjusted R-squared & 0.862530 & S.D. dependent var & 0.154515 \\
S.E. of regression & 0.057289 & Akaike info criterion & - \\
Sum squared resid & 0.180513 & Schwarz criterion & 2.816017 \\
& & & - \\
Log likelihood & 87.07251 & Hannan-Quinn criter. & 2.675167 \\
Durbin-Watson stat & 2.153634 & & - \\
\hline
\end{tabular}

El modelo es construido con los coeficientes que arroja la herramienta de regresión. Para el caso del modelo planteado en esta investigación se expresa de la siguiente forma:

$$
\mathrm{Y}(\mathrm{EXP})=0.9699(\mathrm{IPP})+0.4302(\mathrm{TC})+1.0731(\mathrm{SR})
$$

Los coeficientes son en su mayor parte significativos. Las tres variables, como era esperado, influyen de forma positiva en el nivel de las exportaciones, es así que un incremento de un punto porcentual en los niveles de alguna de las tres variables en consideración incrementa las exportaciones en el siguiente periodo.

Se comprueba la significancia de cada variable haciendo análisis de la tabla \#1 que reporta el modelo, siendo todos los coeficientes significativos. Por lo que se puede concluir que el logaritmo de las exportaciones esta explicado en el largo plazo por el logaritmo de las variables IPP, TC y SR.

Luego, se evalúa la normalidad de los residuos para comprobar la validez de la regresión trazada. En el gráfico \#2, se muestra que en la prueba conjunta los residuos siguen una distribución normal al 0.01 de significancia.

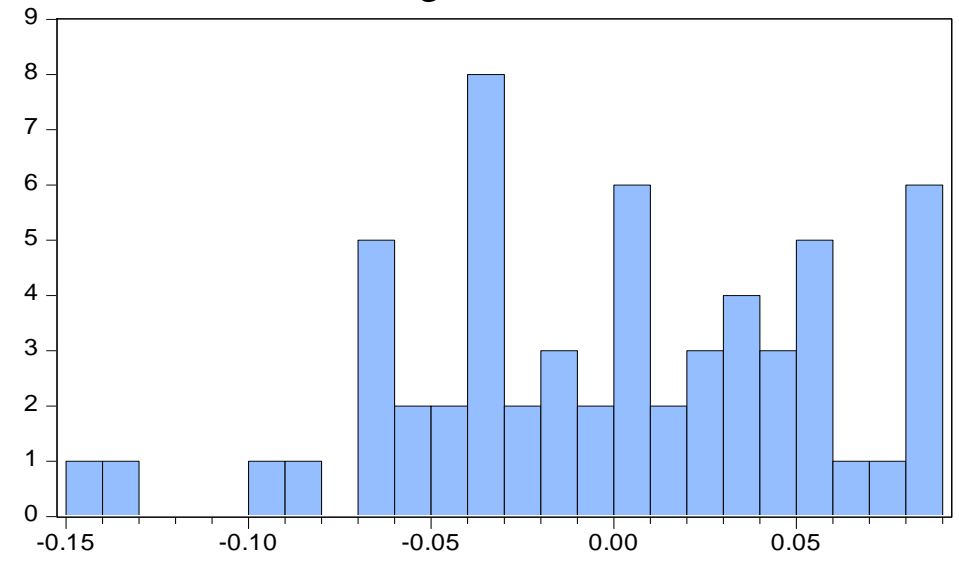

\begin{tabular}{|lr|}
\hline \multicolumn{2}{|l|}{ Series: Residuals } \\
Sample 2010M02 2014M12 \\
Observations 59 \\
Mean & $-9.50 \mathrm{e}-07$ \\
Median & 0.005020 \\
Maximum & 0.088397 \\
Minimum & -0.148313 \\
Std. Dev. & 0.055788 \\
Skewness & -0.396477 \\
Kurtosis & 2.782397 \\
& \\
Jarque-Bera & 1.662145 \\
Probability & 0.435582 \\
\hline
\end{tabular}


Gráfico \#2: Normalidad de los residuos

Por otro lado, en la tabla \#2 se acepta la hipótesis nula de ausencia de auto correlación, para cada uno de los rezagos. Así de la misma forma en la tabla \#3, se realiza una prueba de heteroscedasticidad. La probabilidad de esta prueba es mayor que 0.05 , lo que nos permite aceptar la hipótesis nula, es decir la varianza de los errores es homoscedástica.

Tabla \#2: Prueba de Correlación

\begin{tabular}{|c|c|c|c|c|c|c|}
\hline Autocorrelation & Partial Correlation & & $\overline{\mathrm{AC}}$ & PAC & $\begin{array}{c}\text { Q- } \\
\text { Stat }\end{array}$ & Prob \\
\hline$. * \mid$ &.$* 1.1$ & 1 & & & 1.0580 & 0.304 \\
\hline$. * 1 . \quad \mid$ & $. * \mid$ & 2 & $\begin{array}{r}0.131 \\
-\end{array}$ & $\begin{array}{r}0.131 \\
-\end{array}$ & 2.0044 & 0.367 \\
\hline.$|*|$. &.$\left.\right|^{*}$. & 3 & $\begin{array}{l}0.122 \\
0.132\end{array}$ & $\begin{array}{l}0.142 \\
0.099\end{array}$ & 3.1259 & 0.373 \\
\hline$* *||$. & $* *||$. & 4 & 0.260 & 0.257 & 7.5559 & 0.109 \\
\hline.$||$. &.$||$. & 5 & 0.072 & 0.044 & 7.9004 & 0.162 \\
\hline$.\left.\right|^{*} . \mid$ & .1. & 6 & 0.133 & 0.071 & 9.1019 & 0.168 \\
\hline.$\left.\right|^{*}$. & $.\left.\right|^{*} . \mid$ & 7 & 0.081 & 0.191 & 9.5504 & 0.216 \\
\hline.$|\cdot|$ &.$|\cdot|$ & 8 & 0.016 & & 9.5690 & 0.297 \\
\hline.$||$. &.$||$. & 9 & 0041 & 0.010 & 9.6891 & 0.376 \\
\hline$*|\cdot|$ & $. * \mid$ & $\begin{array}{l}1 \\
0\end{array}$ & 0.115 & $0.116^{-}$ & 10.654 & 0.385 \\
\hline.$||$. &.$||$. & $\begin{array}{l}1 \\
1\end{array}$ & 0.010 & 0.038 & 10.662 & 0.472 \\
\hline$.\left.\right|^{*} \cdot \mid$ &.$||$. & $\begin{array}{l}1 \\
2\end{array}$ & 0.106 & 0.064 & 11.521 & 0.485 \\
\hline$*^{*} . \quad \mid$ & $* *||$. & $\begin{array}{l}1 \\
3\end{array}$ & 0.202 & 0.228 & 14.725 & 0.325 \\
\hline$\left.\cdot\right|^{*} \cdot \mid$ &.$|\cdot|$ & $\begin{array}{l}1 \\
4\end{array}$ & 0.148 & 0.070 & 16.482 & 0.285 \\
\hline$\left.\cdot\right|^{*} \cdot \mid$ & $.\left.\right|^{*} . \quad \mid$ & $\begin{array}{l}1 \\
5\end{array}$ & 0.161 & 0.170 & 18.598 & 0.233 \\
\hline$* *||$. &.$*|. \quad|$ & $\begin{array}{l}1 \\
6\end{array}$ & 0.313 & $0.178^{-}$ & 26.784 & 0.044 \\
\hline.$* 1.1$ & $* *||$. & $\begin{array}{l}1 \\
7\end{array}$ & 0.087 & 0.276 & 27.437 & 0.052 \\
\hline.$|\cdot|$ &.$|\cdot|$ & $\begin{array}{l}1 \\
8\end{array}$ & 0.036 & 0.013 & 27.554 & 0.069 \\
\hline$. * \mid$ &.$|\cdot|$ & $\begin{array}{l}1 \\
9\end{array}$ & 0.125 & 0.026 & 28.970 & 0.066 \\
\hline.$||$. & $. * 1 . \mid$ & $\begin{array}{l}2 \\
0\end{array}$ & 0.011 & $0.161^{-}$ & 28.982 & 0.088 \\
\hline$.\left.\right|^{*} . \mid$ &.$||$. & $\begin{array}{l}2 \\
1\end{array}$ & 0.101 & $\begin{array}{r}- \\
0.054\end{array}$ & 29.952 & 0.093 \\
\hline.$*||$. &.$||$. & $\begin{array}{l}2 \\
2\end{array}$ & $0.094^{-}$ & $\begin{array}{r}- \\
0.058\end{array}$ & 30.810 & 0.100 \\
\hline.$*||$. & $. * \mid$ & $\begin{array}{l}2 \\
3 \\
\end{array}$ & $0.145^{-}$ & $0.145^{-}$ & 32.918 & 0.083 \\
\hline
\end{tabular}




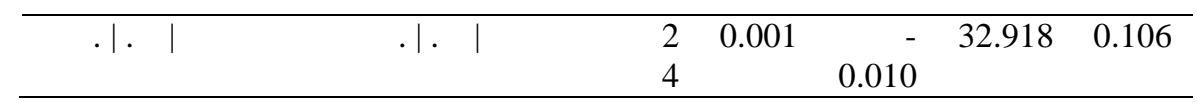

Tabla \#3: Prueba de Heteroscedasticidad

\begin{tabular}{|c|c|c|c|}
\hline \multicolumn{4}{|c|}{ Heteroskedasticity Test: White } \\
\hline F-statistic & 1.480051 & Prob. F(10,48) & 0.1762 \\
\hline Obs*R-squared & 13.90483 & Prob. Chi-Square(10) & 0.1774 \\
\hline Scaled explained SS & 10.76882 & Prob. Chi-Square(10) & 0.3758 \\
\hline
\end{tabular}

Entonces, el modelo cumple con la condición de normalidad, auto correlación y heteroscedasticidad y además los signos de los coeficientes son coherentes con la teoría económica, razones por las cuales se puede aceptar que el modelo planteado explica la relación entre las exportaciones y el índice de precios al productor, tipo de cambio real y salario real.

\section{Conclusiones}

El trabajo de investigación tiene como finalidad establecer la relación de las exportaciones con el precio relativo de los bienes representado por el tipo de cambio real durante el periodo 2000-2014. El trabajo desarrollado ha permitido comprobar la hipótesis del mismo, es decir, que los precios relativos de los bienes determinan a las exportaciones ecuatorianas.

Asimismo, el modelo econométrico permite corroborar que las variables que incluye el modelo reducido planteado por Mesa, Cock y Jiménez (1999), son aquellas que guardan una relación con las exportaciones.

En efecto el resultado obtenido de la aplicación del modelo permite confirmar que las variables económicas como el salario real, tipo de cambio real e índice de precios al productor tienen estrecha relación con el comportamiento de las exportaciones en el Ecuador.

Las variables utilizadas son reales y han sido transformadas previamente en logaritmos para facilitar su interpretación. A través de la aplicación de una regresión linear múltiple, se comprobó que las variables utilizadas para el análisis tienen estrecha relación con las exportaciones ecuatorianas. Los resultados permiten armar el modelo el cual permite explicar el comportamiento de las exportaciones ecuatorianas con respecto a las variables IPP, TC y SR.

Si se analiza el modelo con respecto a la hipótesis que se plantea en la investigación, el precio relativo de bienes representado por el tipo de cambio real tiene un efecto representativo sobre la dinámica de las exportaciones, es así que una variación de un punto porcentual en el tipo de cambio real afecta positivamente a las exportaciones en cuarenta y tres puntos aproximadamente. Por lo tanto, el modelo explica la relación que existe entre los precios relativos de los bienes y las exportaciones aplicadas para el caso de Ecuador.

Los resultados del estudio demuestran que un mayor incentivo en las variables empleadas tendrá un efecto positivo en las exportaciones, razón por la cual es necesario mejorar las condiciones para el mejor desempeño de las exportaciones. En las variables en las cuales se pueden mejorar internamente como país son los salarios de esta manera se mejorará la 
productividad y por ende se desarrollará el sector exportador, como se evidencia en el modelo explicado.

Finalmente, es importante analizar los efectos que tienen otro tipo de variables sobre las exportaciones y en general sobre la balanza comercial del Ecuador, por lo que se deja abierta la posibilidad para fututos trabajos investigativos de examinar la importancia de los muchas más variables explicativas sobre el comercio ecuatoriano.

Además, este trabajo de investigación puede ser utilizado como un estudio de referencia que ayude al análisis de las actividades comerciales y económicas encaminadas a dinamizar el mercado y la oferta exportable, generando estrategias que permitan impulsar la integración económica del Ecuador, tomando como eje fundamental las exportaciones. Asimismo, el tema de investigación planteado da la pauta para entender de mejor manera el desenvolvimiento y evolución de las exportaciones en el país, analizando sus principales variables económicas determinantes y observar como estos influyen en el rubro de la balanza comercial ecuatoriana.

\section{Bibliografía}

Agosin, M. R.-O. (2012). Determinants of export diversification around the world: 1962-2000. The World Economy, 295-315.

Aguirre, M. G., \& Chávez, J. C. (2014). Relación de causalidad entre el índice de precios del productor y el índice de precios del consumidor incorporando cambios estructurales El caso de México. Contaduría y Administración, 179-196.

Akçay, S. (2011). The causal relationship between producer price index and consumer price index: Empirical evidence from selected European countries. International Journal of Economics and Finance, 227.

Alarco Tosoni, G. (2011). Exportaciones, tipo de cambio y enfermedad holandesa: el caso peruano. Investigación económica, 115-143.

Aller, R. A., Sáinz, A. F., \& Sáenz, B. G. (2002). Precios relativos y exportaciones manufactureras españolas: ¿explotan las empresas industriales una cadena de valor multinacional? Universidad de Chile.

Almeida, P. (2005). Elementos para el Cálculo del Tipo de Cambio Real en el Ecuador. Notas Tecnicas del Banco Central.

Arévalo, J. L., Lima, J. R., \& Araújo, A. F. (2013). Determinantes de la oferta de exportación de mango: estudio de caso para el Perú. Revista de Economia e Sociologia Rural, s101-s120.

Asiain, A. (2010). Tipo de cambio, precios internacionales y retenciones en un modelo estructuralista de corto plazo. Economía, 57-78.

Báez P, D. S. (2014). Comercio Internacional: Un breve análisis desde Ecuador enfocado en los países en vías de desarollo. Observatorio de la Economía Latinoamericana. 
Bajo Rubio, O. (1996). Teorías del comercio internacional: una panorámica. Ekonomiaz: Revista vasca de economía, 12-27.

Balaguer, J., Orts, V., \& Uriel, E. (2000). Comportamiento de precios en la industria europea de pavimentos y revestimientos cerámicos. ICE: Revista de Economía, 1-25.

Banco Central del Ecuador. (2015). Boletin Estadistico Mensual.

Berdún Chéliz, P. (2002). La teoría y la práctica de la estrategia de industrialización orientada hacia la exportación. Acciones e Investigaciones Sociales, 71-88.

Berdún Chéliz, P. (2011). La teoría y la práctica de la estrategia de industrialización orientada hacia la exportación. Acciones e Investigaciones Sociales, 71-88.

Berrettoni, D., \& Castresana, S. (2007). Exportaciones y tipo de cambio real: el caso de las manufacturas industriales argentinas. Revista del CEI: comercio exterior e integración.

Blanco, R. G. (2011). Diferentes teorías del comercio internacional. ICE: Revista de economía, 103 118.

Cáceres, L. R. (2007). Exportaciones, inversión y crecimiento económico en Centroamérica. El Trimestre Económico, 719-743.

Camacho, D. D. (2011). Relación empírica entre la productividad y las exportaciones manufactureras mexicanas, 2000-2008. Análisis Económico, 69.

Camacho, D. D. (2011). Relación empírica entre la productividad y las exportaciones manufactureras mexicanas, 2000-2008. Análisis Económico, 69.

Cardozo, P. P., Chavarro, A., \& Ramírez, C. A. (2007). Teorías de internacionalización. Panorama. Cueavas-Ahumada, V. M. (2012). México: dinámica de las exportaciones manufactureras. Revista de la CEPAL, 153-174.

Cuevas-Ahumada, V. M. (2011). Determinantes de las exportaciones manufactureras en Argentina y México: un estudio comparativo. Economía, sociedad y territorio, 121-159.

Davis, D. R. (1995). Intra-industry trade: a Heckscher-Ohlin-Ricardo approach. Journal of international Economics, 201-226.

De la Cruz Gallegos, J. L., Rivera, C. C., \& Castro, P. G. (2009). Economic growth, foreign direct investment and international trade: evidence on causality in the Mexican economy. Revista Brasileira de Economía de Empresas, 17.

De la Rosa Mendoza, J. R. (2006). Dos enfoques teóricos sobre el proceso de crecimiento económico: con énfasis en las exportaciones manufactureras. Análisis Económico, 93-119.

Díaz-Alejandro, C. (1977). Efectos de las exportaciones no tradicionales en la distribución del ingreso: el caso colombiano. El Trimestre Economico, 411-426. 
Doherty, M. P. (2012). Behavior of the Producer Price Index in a Global Economy. The Monthly Labor Review, 135.

Freire, M. B. (1997). Determinantes de las exportaciones no tradicionales en el Ecuador 1976-1995. Banco Central del Ecuador.

Gómez Chiñas, C., \& Camacho Erazo, R. (2011). Las exportaciones mexicanas de manufacturas. Análisis de cointegración con respecto a sus factores determinantes. Ecorfan Journal, 1-24.

Haghnejad, A., Mehrara, M., Meybodi, F. J., \& Dehnavi, J. A. (2010). Foreign direct investment, exports, and economic growth in the developing countries: a panel data approach. Journal of Academic Research in Economics, 259-280.

Iglesias, R. (2005). El rol del tipo de cambio real y la inversión en la diversificación de exportaciones en América Latina y el Caribe. CEPAL.

Lanteri, L. N. (2014). Tipo de cambio real efectivo y exportaciones de manufacturas no tradicionales. Evidencia para Argentina. Revista Latinoamericana de Desarrollo Económico, 117-136.

Loza Tellería, G. (2000). Tipo de cambio, exportaciones e importaciones: el caso de la economía boliviana. Revista de Análisis del Banco Central de Bolivia, 7.

Mankiw, N. G. (2005). Macroeconomía. (A. Bosch, Ed.)

Mesa Parra, F., Cock, M. I., \& Jiménez, A. P. (1999). Evaluación teórica y empírica de las exportaciones no tradicionales en Colombia. Revista de Economía del Rosario.

Morón Cárdenas, J. A. (2002). Los determinantes estructurales de las exportaciones departamentales en Colombia.

Rodríguez Benavides, D., \& López Herrera, F. (2010). Exportaciones y productividad laboral del sector manufacturero en México. Problemas del desarrollo, 41-56.

Uquillas, C. A. (2008). El modelo económico industrial en el Ecuador. Observatorio de la Economía Latinoamericana.

Vos, R., \& León, M. (2003). Unidad de Información y Análisis--SIISE--de la Secretaría Técnica del Frente Social.

Zarzoso, I. M., \& Horsewood, N. J. (2004). Exportaciones relativas España-Reino Unido a los países de la OCDE: precios, gustos y calidades. Información Comercial Española. ICE: Revista de economía, 29-38.

Zhang, X., \& Liu, X. (2012). How responsive are Chinese exports to exchange rate changes? Evidence from firm-level data. The Journal of Development Studies, 1489-1504. 